\title{
A Three-Dimensional Computational Model of Action Potential Propagation Through a Network of Individual Cells.
}

\author{
Pierre-Elliott Bécue ${ }^{1,2,3}$, Mark Potse ${ }^{1,2,3}$, Yves Coudière ${ }^{1,2,3}$ \\ ${ }^{1}$ INRIA Bordeaux Sud-Ouest, Talence, France \\ ${ }^{2}$ Institut de Mathématiques de Bordeaux, Talence, France \\ ${ }^{3}$ Institut de Rythmologie et Modélisation Cardiaque, Pessac, France
}

\begin{abstract}
It has been suggested in the literature that a disorganisation of cardiac tissue at the cellular scale may affect the propagation of the action potential (AP) at the tissue scale, and may play a role in arrhythmia.

We developed a model of the myocardium at sub-cellular resolution in which the intracellular space, the cell membrane, and the extracellular space are discretized individually. We tested this approach on small, hand-crafted twoand three-dimensional networks of cells.
\end{abstract}

\section{Introduction}

The standard bidomain or monodomain equations model a cardiac tissue at the macroscopic scale. They may be derived by homogenization of microscopic bidomain equations, which model the propagation of the cardiac action potential (AP) on a network of individual cells. For homogenization, the network is assumed to be periodic.

Yet, dysfunction or disorganization (non periodicity) of the tissue at the cellular level may affect the propagation of the AP at the tissue scale. For instance, zigzag propagation at a cellular level has been hypothesized to lead to arrhythmias [1]. These alterations cannot be represented by the homogenized bidomain or monodomain models.

To understand and tackle such questions, we plan to simulate the bidomain equations written at the cellular scale on a manually designed network of cells. The equations were first studied theoretically [2], and we proved existence of a solution (see ref.[3]).

In this paper we recall the equations and their properties, describe a numerical method to compute approximate solutions, and show some first results obtained on a set of 1 to 3 cells. The model, its limitations, and possible improvements are discussed.

\section{The bidomain model at the cell scale}

The geometry and associated notations are given in fig. 1. The microscopic equations are, for all time $t>0$,

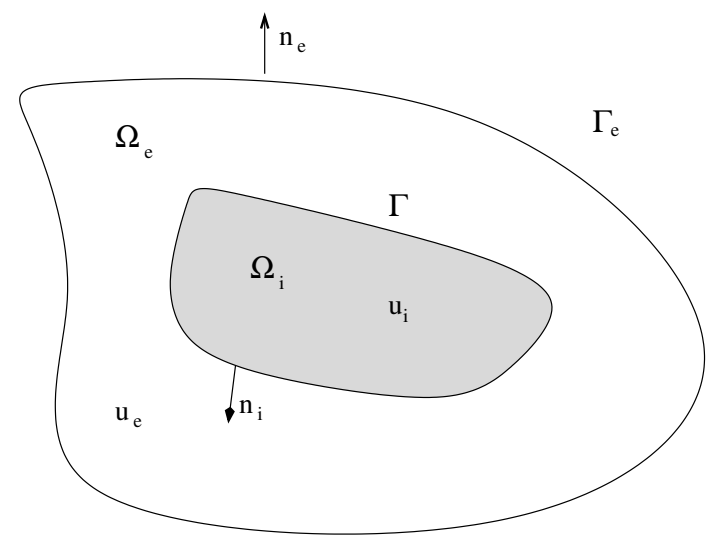

Figure 1. The model comprises an intracellular domain $\Omega_{\mathrm{i}}$, a cell membrane $\Gamma$, and an extracellular domain $\Omega_{\mathrm{e}}$. The domain boundary is denoted as $\Gamma_{\mathrm{e}}$.

$-\nabla \cdot\left(\sigma_{\mathrm{i}} \nabla u_{\mathrm{i}}\right)=0$ in the intracellular domain $\Omega_{\mathrm{i}}$ and $-\nabla \cdot\left(\sigma_{\mathrm{e}} \nabla u_{\mathrm{e}}\right)=0$ in the extracellular domain $\Omega_{\mathrm{e}}$. They are completed by the transmission conditions

$$
-\sigma_{\mathrm{i}} \nabla u_{\mathrm{i}} \cdot n_{\mathrm{i}}=\sigma_{\mathrm{e}} \nabla u_{\mathrm{e}} \cdot n_{\mathrm{e}}=c_{m} \partial_{t} v+I_{\mathrm{ion}}(v, w)
$$

on the cell membrane $\Gamma$, and by the no-flux boundary condition $\sigma_{\mathrm{e}} \nabla u_{\mathrm{e}} \cdot n_{\mathrm{e}}=0$ on the external boundary $\Gamma_{\mathrm{e}}$. Here, $u_{\mathrm{i}, \mathrm{e}}$ denote the intra- and extracellular potential fields, and $\sigma_{\mathrm{i}, \mathrm{e}}$ are scalar electrical conductivities in the intraand extracellular domains. The trans-membrane voltage is $v=u_{\mathrm{i}}-u_{\mathrm{e}}$, defined only on the interface $\Gamma$. The cell membrane is modeled by the conductance $c_{m}$ in parallel with an ionic current $I_{\text {ion }}$. The vector $w$, defined on the interface $\Gamma$, gathers the additional state variables of the ionic model. It solves a differential equation of the form $\partial_{t} w=H(v, w)$, depending on the ionic model chosen. We assume that initial data are given only for the trans-membrane voltage $v$, as $v(0, x)=v^{0}(x)$ for $x \in \Gamma$. 
There exists a well posed weak formulation of these equations, where $u_{\mathrm{i}}$ and $u_{\mathrm{e}}$ are searched in the spaces $H^{1}\left(\Omega_{\mathrm{i}, \mathrm{e}}\right)$, with an additional gauge condition for sake of uniqueness. The functions $u_{\mathrm{i}}$ and $u_{\mathrm{e}}$ must solve:

$$
\begin{gathered}
\int_{0}^{T} \int_{\Omega_{\mathrm{i}}} \sigma_{\mathrm{i}} \nabla u_{\mathrm{i}} \cdot \nabla \varphi_{\mathrm{i}} \mathrm{dxd}+\int_{0}^{\mathrm{T}} \int_{\Omega_{\mathrm{e}}} \sigma_{\mathrm{e}} \nabla \mathrm{u}_{\mathrm{e}} \cdot \nabla \varphi_{\mathrm{e}} \mathrm{dxdt} \\
-\int_{0}^{T} \int_{\Gamma} c_{m} v \partial_{t} \tilde{\varphi} \mathrm{dxd}+\int_{0}^{\mathrm{T}} \int_{\Gamma} \mathrm{I}_{\mathrm{ion}}(\mathrm{v}) \tilde{\varphi} \mathrm{dxd} \mathrm{t} \\
+\int_{\Gamma} c_{m} v^{0} \tilde{\varphi}(t=0) \mathrm{dx}=0
\end{gathered}
$$

for all test functions $\varphi_{\mathrm{i}}$ and $\varphi_{\mathrm{e}}$ in $H^{1}\left(\Omega_{\mathrm{i}, \mathrm{e}}\right)$, and with $\tilde{\varphi}=$ $\varphi_{\mathrm{i}}-\varphi_{\mathrm{e}}$, defined on the cell membrane $\Gamma$.

We approximate the solution with the P1-Lagrange finite element method, and use an Euler time-stepping method, implicit on the diffusion terms, and explicit on the ionic ones. Hence, for a sequence of times $t^{n}=n \delta t$ $(\delta t>0)$, we solve the following discrete equations on $u_{\mathrm{i}}^{n}$ and $u_{\mathrm{e}}^{n}$ in the discrete P1 spaces:

$$
\begin{gathered}
\int_{\Omega_{\mathrm{i}}} \sigma_{\mathrm{i}} \nabla u_{\mathrm{i}}^{n} \cdot \nabla \varphi_{\mathrm{i}} \mathrm{dx}+\int_{\Gamma}\left[\mathrm{c}_{\mathrm{m}} \frac{\mathrm{v}^{\mathrm{n}}}{\delta \mathrm{t}}+\mathrm{I}_{\mathrm{ion}}\left(\mathrm{v}^{\mathrm{n}-1}\right)\right] \varphi_{\mathrm{i}} \mathrm{ds} \\
=\int_{\Gamma} c_{m} \frac{v^{n-1}}{\delta t} \varphi_{\mathrm{i}} \mathrm{ds}, \\
\int_{\Omega_{\mathrm{e}}} \sigma_{\mathrm{e}} \nabla u_{\mathrm{e}}^{n} \cdot \nabla \varphi_{\mathrm{e}} \mathrm{dx}-\int_{\Gamma}\left[\mathrm{c}_{\mathrm{m}} \frac{\mathrm{v}^{\mathrm{n}}}{\delta \mathrm{t}}+\mathrm{I}_{\mathrm{ion}}\left(\mathrm{v}^{\mathrm{n}-1}\right)\right] \varphi_{\mathrm{e}} \mathrm{ds} \\
=-\int_{\Gamma} c_{m} \frac{v^{n-1}}{\delta t} \varphi_{\mathrm{e}} \mathrm{ds},
\end{gathered}
$$

where we set $v^{n}=u_{\mathrm{i}}^{n}-u_{\mathrm{e}}^{n}$ for $x \in \Gamma$. This requires that the meshes of the domains $\Omega_{\mathrm{i}}$ and $\Omega_{\mathrm{e}}$ coincide on the boundary $\Gamma$.

For the first tests described below, we used the MitchellSchaeffer (MS) model because it is computationally simple, and helps to have a qualitative understanding of the results.

Finally, for each time step, we have to solve a linear system of equations of the form $A U^{n}=F^{n}$ where $U^{n}=$ $\left(\begin{array}{ll}U_{\mathrm{i}}^{n} & U_{\mathrm{e}}^{n}\end{array}\right)^{T}$ is the vector of the degrees of freedom of $u_{\mathrm{i}}^{n}$ and $u_{\mathrm{e}}^{n}$, and the right-hand side vector $F^{n}=\left(\begin{array}{ll}F_{\mathrm{i}}^{n} & F_{\mathrm{e}}^{n}\end{array}\right)^{T}$ involves the nonlinear function $I_{\text {ion }}$. In addition, we compute the Euler approximation of the degrees of freedom $W$ (for the function $w$ ) as $W^{n}=W^{n-1}+\delta t H\left(V^{n}, W^{n}\right)$ on $\Gamma$, with $W^{n}=U_{\mathrm{i}}^{n}-U_{\mathrm{e}}^{n}$.

\section{Simulations and results}

\subsection{Specificities of the linear system}

The matrix of the linear system is symmetric and writes, by blocks, as:

$$
A=\left(\begin{array}{ll}
A_{\mathrm{ii}} & A_{\mathrm{ie}} \\
A_{\mathrm{ei}} & A_{\mathrm{ee}}
\end{array}\right)
$$

We can see from the discrete weak formulation above that the stiffness matrix must be assembled on the whole volume, while the mass matrix is assembled only on the points that belong to the trans-membrane interface $\Gamma$. The terms corresponding to the coupling between the intra- and extracellular potential fields belong to the matrices $A_{\mathrm{ei}}$ and $A_{\mathrm{ie}}$. It is challenging to compute efficiently these matrices in our finite-element code, CEPS. In practice, it was decided to split a global mesh into two sub-meshes (intraand extracellular domains) with coupling data on the cell membrane nodes.

In addition, the initial problem is a pure Neuman equation (no-flux condition on the external boundary). Hence the linear system has a solution defined up to a constant. Here we compute the solution perpendicular to the kernel space of the matrix $A$ (corresponding to a gauge condition) with a conjugate gradient iterative solver, as suggested in ref. [4].

\subsection{Computational solver and meshes}

Our model was programmed in the software code CEPS developed at Inria. It relies on the PETSc library to solve the linear systems on parallel computers. In test cases below, the cell membrane geometry was designed manually (using Blender, or gmsh) as a triangulated surface, inside a rectangular box. A volume mesh was then computed using tetgen. For the three-dimensional simulations, the intracellular mesh has around $43 \mathrm{k}$ points, $270 \mathrm{k}$ elements (tetrahedra plus triangles). The extracellular mesh has around $171 \mathrm{k}$ points and $1 \mathrm{M}$ elements. Figure 2 shows the surface mesh (cell membrane) for three cells that are connected through two transverse surfaces.

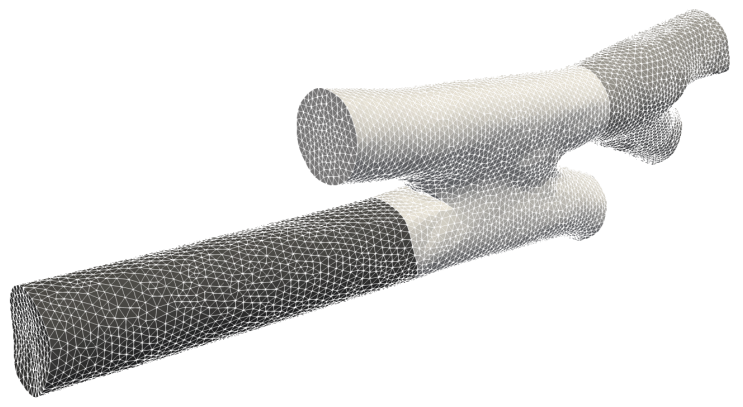

Figure 2. The three dimensional triangulated cell surface.

\subsection{A two-dimensional study}

We consider one or two rectangular cells of size $100 \times$ $20 \mu \mathrm{m}$ inside a box of size $150 \times 40 \mu \mathrm{m}(250 \times 40$ for 2 cells). An AP is initiated by applying a current between a small patch on the left (anode) of the extracellular domain, and a larger one on the right (cathode). The ratio of surface 
between the cathode and the anode is 10 . The average of the electrical potential on the domain remains zero. With such a stimulation, an AP is expected to appear on the left side, and to propagate to the right. The intra- and extracellular potential fields are represented on the figures below between -1 (blue) and +1 (red, arbitrary unit, related to the MS model). The initial data are $u_{\mathrm{i}}=0, u_{\mathrm{e}}=0$, and $w=1$ (resting state of the MS model).

For one cell (fig. 3), we apply a total current of $I=4.6$ at $t=0.1 \mathrm{~ms}$, during $0.02 \mathrm{~ms}$. The first image shows an almost linear electrical fields due to the current applied between the anode and the cathode, with a jump at the membrane interface, due to the jump of electrical conductivity. After the stimulation, the potential falls a little, and the ionic model dynamics alters the jump, thus leading to depolarizing cell at $t=0.4 \mathrm{~ms}$ (second image). The last image shows an almost fully depolarized cell at $t=5.0 \mathrm{~ms}$. After approximately $250 \mathrm{~ms}$, the resting state is restored (not displayed here). While not shown here, the propagation of the potential along the cell takes around $0.2 \mathrm{~ms}$, which is faster than the depolarization of the cell which takes several milliseconds.

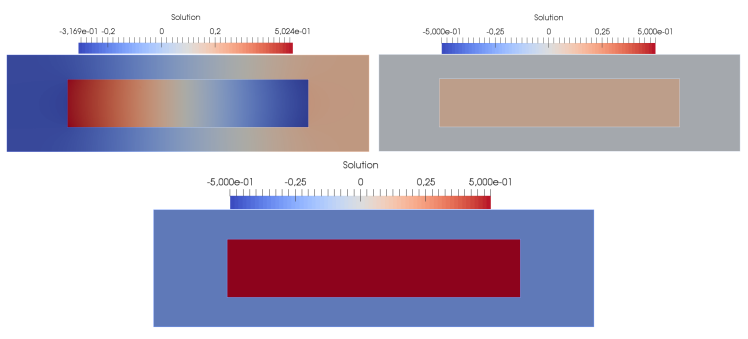

Figure 3. Potential fields at $t=0.15 \mathrm{~ms}$ (left) and $t=$ $0.4 \mathrm{~ms}$ (right) and $t=5.0 \mathrm{~ms}$ (bottom)

Fig. 4 shows a setup with two cells connected by a channel of size $10 \times 2 \mu \mathrm{m}$. We apply a total current of $I=11.0$ at $t=0.1 \mathrm{~ms}$ during $0.02 \mathrm{~ms}$. Like for one cell, the left cell is reacting first (first image), and then the potential crosses to the right cell (second image). The last image shows the two cells almost depolarized. A small delay is due to the presence of the channel that connects the two cells. It mimics a gap-junction channel. The cells return to the resting state in $250 \mathrm{~ms}$ as in the previous test case. In this specific simulation, one can observe that the AP is crossing the channel in the first image. In practice, the potential takes around $0.2 \mathrm{~ms}$ to propagates from the first cell to the second. As the first cell continues to depolarize, the balancing process that leads both cells to reach synchronized potential takes between $0.4 \mathrm{~ms}$ and $0.6 \mathrm{~ms}$.

\subsection{A three-dimensional case}

A three-dimensional test case was designed using the surface mesh shown in fig. 2. The three cells in this ge-

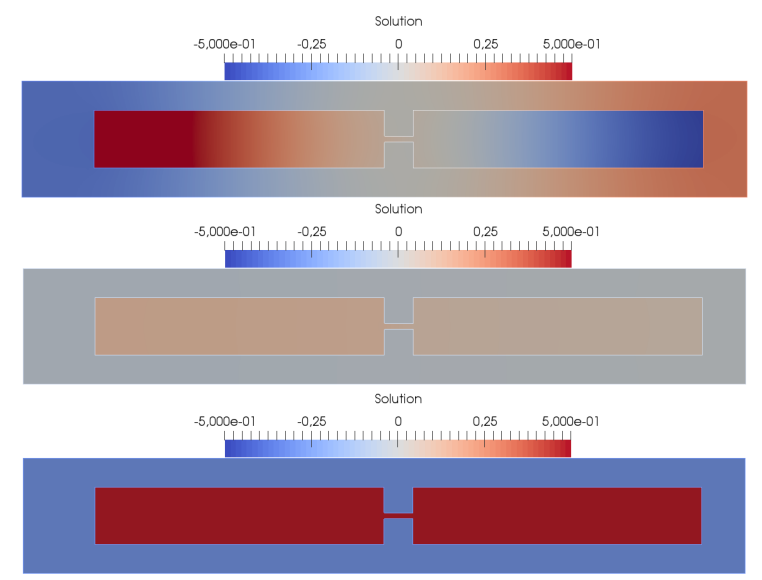

Figure 4. Potential fields at $t=0.15 \mathrm{~ms}$ (top) and $t=$ $0.4 \mathrm{~ms}$ (middle) and $t=5.0 \mathrm{~ms}$ (bottom) on the two cells test.

ometry are not separated one from another like in the twodimensional case, and hence the small channels (mimicking the gap-junctions) do not exist. Instead they are connected through their transverse surface. In the simulation presented here, there is no model on these surfaces, and they behave like transparent interfaces between cells (continuity of the potential field and of the normal flux). The MS model was applied on the interfaces that separate the intra- and extracellular domains. The conductivity ratio between the extra- and the intracellular media was set to 2 , and the total size of the domain is of the order of $300 \times 100 \times 70 \mu \mathrm{m}$.

Two volumes are defined, on the left side (cathodal volume), and on the right side (anodal volume) of the extracellular domain. The ratio between the volumes is set to 3.5 , and a current of is applied through these volumes. The initial data are $u_{\mathrm{i}}=0, u_{\mathrm{e}}=0$, and $w=1$ (resting state of the MS model), and the total current is $I=16300$, still using an arbitrary unit because of the MS model.

Figure 5 shows one snapshot at $t=40 \mathrm{~ms}$ of the electrical field on a slice of the three-dimensional domain (same scaling as above). One observes the action potential that almost crossed the three cells. On the whole, the threedimensional simulation happens alike the two previous, with small differences because of it's volumic properties. The trans-membrane voltage $v=u_{\mathrm{i}}-u_{\mathrm{e}}$ during the depolarization has been recorded at the 4 points displayed on fig. 5, denoted up right (red), middle (blue), bottom (green), and bottom left (purple), along with a reference simulation (black) of such voltage in one dimension (using finite difference method). They are displayed in fig. 6 , where the propagation from right to left is almost invisible after the stimulus. These curves differ only from the onedimensional one because of the absence of diffusion in the latter case. 


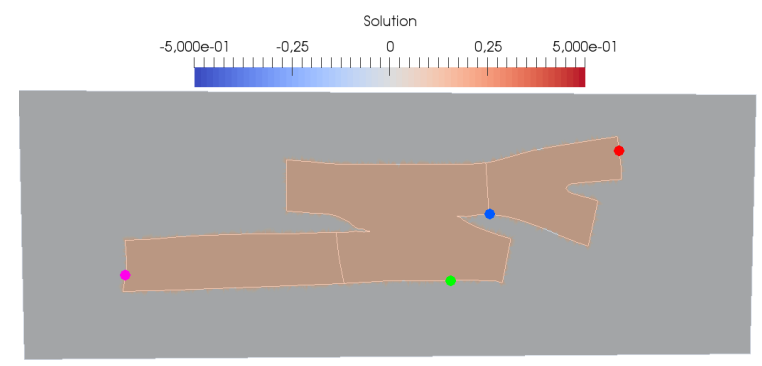

Figure 5. Potential fields at $t=0.4 \mathrm{~ms}$

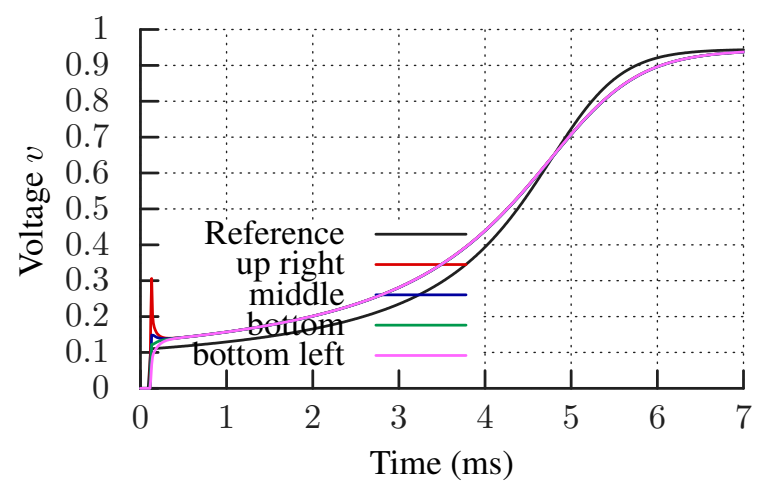

Figure 6. Voltage $v$ on the four points displayed on figure 5, and reference ODE solution of the MS model.

\section{Discussion}

In the original derivation of the bidomain equations by Krassovska \& Neu [5], the cells are not connected end-toend to each other by intercalated discs, but by small channels that model the gap-junctions. In the two-dimensional cases, we use the geometrical setup of the article [5]. We can observe a slower propagation through the channel. But the geometry is not coherent with the actual connections between cells at intercalated discs. In the threedimensional case, we have a better geometry. But then a transmission condition must be imposed between two connected cells, according to a gap junction model. Our idea is to complete the equations with the condition $-\sigma_{\mathrm{i}, 1} \nabla u_{\mathrm{i}, 1}$. $n_{\mathrm{i}, 1}=\sigma_{\mathrm{i}, 2} \nabla u_{\mathrm{i}, 2} \cdot n_{\mathrm{i}, 2}=g\left(u_{\mathrm{i}, 1}-u_{\mathrm{i}, 2}\right)\left(u_{\mathrm{i}, 1}-u_{\mathrm{i}, 2}\right)$, along the intercalated disc that separates cells 1 and 2 . The gap-junction conductance $g\left(u_{\mathrm{i}, 1}-u_{\mathrm{i}, 2}\right)$ might be linear or non linear, or even have its own dynamics, modeled as in ref. [6].

From a numerical and mathematical standpoint, geometries with channels like in the two-dimensional test cases create singularities in the solutions (near corners), where numerical instabilities may develop, and necessitate very fine meshes to discretize the channels and spaces between cells. The alternative, more general, solution described above is quite complex to implement in a general manner, so as to be able to scale the simulations to tens, hundreds or thousands of cells. It is currently being implemented in CEPS. Additional difficulties are expected for the larger number of cells (mesh size, memory requirement, condition number of the linear system, computational time).

As for the velocities, an AP going at $50 \mathrm{~cm} / \mathrm{s}$ should travel $100 \mu \mathrm{m}$ in $0.2 \mathrm{~ms}$. On these test cases, the considered domain is too small and nothing confirming this velocity can be observer. A test with a domain measuring at least 10 cells is mandatory to investigate further.

\section{Conclusion}

A solver of the microscopic model has been designed. Some preliminary results have been computed in simple two-dimensional and three dimensional networks of cells. The biophysical impact of the results is currently limited by the simplicity of the cases. We expect results concerning larger two-dimensional networks of cells and simplified end-to-end cell connections to address relevant questions regarding the propagation of AP. After the implementation and numerical difficulties are overcome, we also expect results with gap-junction mathematical models over intercalated discs.

\section{References}

[1] de Bakker JM, van Capelle FJ, Janse MJ, Tasseron S, Vermeulen JT, de Jonge N, Lahpor JR. Slow conduction in the infarcted human heart. 'zigzag' course of activation. Circulation 1993;88(3):915-926. ISSN 0009-7322.

[2] Colli-Franzone P, Savaré G. Degenerate Evolution Systems Modeling the Cardiac Electric Field at Micro- and Macroscopic Level. Basel: Birkhäuser Basel. ISBN 978-3-03488221-7, 2002; 49-78.

[3] Bécue PE, Caro F, Potse M, Coudière Y. Theoretical and Numerical Study of Cardiac Electrophysiology Problems at the Microscopic Scale. SIAM Conference on the Life Sciences (LS16), July 2016. URL https://hal.inria.fr/hal-01405837. Poster.

[4] Bochev P, Lehoucq RB. On the finite element solution of the pure neumann problem. SIAM Review 2005;47(1):50-66.

[5] Neu JC, Krassowska W. Homogenization of syncytial tissues. Crit Rev Biomed Eng 1993;21:137-199.

[6] Coudière Y, Davidovic A, Poignard C. The modified bidomain model with periodic diffusive inclusions. In Computing in Cardiology Conference (CinC). 2014; 1033-1036.

Address for correspondence:

Pierre-Elliott Bécue

Maison de la Simulation USR 3441, Bâtiment 565 - Digiteo, CEA Saclay, 91191 Gif-sur-Yvette cdex, France pierre-elliott.becue@inria.fr 